\title{
Análisis crítico de un artículo: ¿Podemos descartar la presencia de hemorragia subaracnoidea con la combinación de tomografía computada cerebral y punción lumbar negativas?
}

\author{
Cynthia Zavala G ${ }^{1, a}$, Carlos Aravena $L^{1,2,3}$. \\ Critically appraised article: \\ Is the combination of negative computed \\ tomography result and negative lumbar \\ puncture result sufficient to rule out \\ subarachnoid hemorrhage? \\ Ann Emerg Med 2008; 51 (6): 707-713 \\ Perry J, Spacek A, Forbes M, Wells G, \\ Mortensen $G$, Symington $C$, Fortin N, Stiell N.
}

\begin{abstract}
Study objective: Current clinical practice assumes a negative computed tomography (CT) head scan result and a negative lumbar puncture result together are adequate to rule out subarachnoid hemorrhage in patients with acute headache. Our objective is to determine the sensitivity of a negative CT result combined with a negative lumbar puncture result to exclude subarachnoid hemorrhage. Methods: This prospective cohort study was conducted at 2 tertiary care emergency departments (EDs) during 3 years. We enrolled all patients who were older than 15 years, had a nontraumatic acute headache and normal neurologic examination result, and who had a CT head scan and a lumbar puncture if the CT result was negative (ie, no blood in the subarachnoid space). Patients were followed up with a structured telephone questionnaire 6 to 36 months after their ED visit and electronic hospital records review to ensure no missed subarachnoid hemorrhage. We calculated sensitivity, specificity, and likelihood ratios of the strategy of CT and then lumbar puncture for subarachnoid hemorrhage. Results: Five hundred ninety-two patients were enrolled, including 61 with subarachnoid hemorrhage. The mean patient age was 43.6 years, with $59.1 \%$ female patients. All cases of subarachnoid hemorrhage were identified on initial CT or lumbar puncture. One patient without subarachnoid hemorrhage was subsequently diagnosed with cerebral aneurysm, requiring surgery. The strategy classified patients with subarachnoid hemorrhage with sensitivity, specificity, and positive and negative likelihood ratios (with 95\% confidence intervals [CIs) of 100\% (95\% CI 94\% to 100\%), 67\% (95\% CI $63 \%$ to $71 \%$ ), 3.03 (95\% CI 2.69 to 3.53), and 0. For diagnosis of subarachnoid hemorrhage or aneurysm, these were $98 \%$ ( $95 \%$ CI $91 \%$ to $100 \%$ ), 67\% (95\% CI 63\% to 71\%), 2.98 (95\% CI 2.63 to 3.38), and 0.02 (95\% CI 0.00 to 0.17$)$, respectively. Conclusion: To our knowledge, this is the largest prospective study evaluating the accuracy of a strategy of CT and lumbar puncture to rule out subarachnoid hemorrhage in alert ED patients with an acute headache. This study validates clinical practice that a negative CT with a negative lumbar puncture is sufficient to rule out subarachnoid hemorrhage.
\end{abstract}

\footnotetext{
${ }^{1}$ Unidad de Medicina Basada en Evidencia, Pontificia Universidad Católica de Chile.

${ }^{2}$ Departamento de Medicina Interna, Pontificia Universidad Católica de Chile.

${ }^{3}$ Servicio de Medicina, Hospital Dr. Sótero del Río.

aesidente de Psiquiatría, Universidad de Los Andes

Correspondencia a: Dr. Carlos Aravena. E mail: caaraven@uc.cl
}

MEDICINA BASADA 


\section{CONCLUSIÓN DE LOS REVISORES:}

En pacientes que consultan en un Servicio de Urgencia por cefalea de inicio agudo, la evaluación con tomografía computada cerebral (TCC) y punción lumbar (PL) permitirían descartar la hemorragia subaracnoidea (HSA) como etiología de la cefalea.

\section{Pregunta CĹNICA:}

En pacientes que consultan en Urgencia por cefalea de inicio agudo no traumático, ès suficiente la evaluación con TCC y PL para descartar una HSA?

\section{ConTeXto:}

La HSA se define como la presencia de sangre en el espacio subaracnoideo y es una causa grave de cefalea de inicio agudo entre los consultantes en Servicios de Urgencia. Su incidencia anual se estima en 1/10.000 personas en EE.UU. de Norteamérica, y su prevalencia en pacientes consultantes en servicio de urgencia es de $1 \%{ }^{1}$. Habitualmente los pacientes consultan por un cuadro de cefalea muy intensa, de inicio agudo, sin antecedentes de trauma ni déficit neurológico asociados. Hasta $40 \%$ de los pacientes que presentan una HSA, tienen el antecedente de haber presentado cefalea, consecuencia de pequeños sangrados, denominadas "cefaleas centinela"1-3.

En la práctica habitual, el diagnóstico de HSA se descarta con una TCC sin sangre en el espacio subaracnoideo, sin embargo alrededor de $10 \%$ de los pacientes que presentan HSA tienen un TCC negativo; es este grupo en quienes se considera la realización de una PL para descartar con mayor certeza el diagnóstico ${ }^{1,4}$, evitando la necesidad de angiografía cerebral.

\section{CARACTERÍSTICAS DEL ESTUdio:}

Estudio multicéntrico de cohorte prospectivo, realizado en los Servicios de Urgencia de dos hospitales terciarios de Canadá.

Pacientes: 592 pacientes consecutivos, mayores de 15 años, consultantes en urgencia por cefalea aguda no traumática y sin alteraciones en el examen neurológico. Criterios de exclusión: Cefaleas recurrentes ( $>3$ episodios semejantes en los últimos 6 meses); HSA confirmada, antecedentes de HSA, reevaluación de un cuadro que hubiese consultado previamente, tumores cerebrales, alteraciones al examen neurológico, cefalea post PL.

Intervención: A todos se los evaluó con TCC, y en quienes ésta fue negativa para HSA se realizó una PL Además se siguió a los pacientes con ambos tests negativos por un período de entre 6 y 36 meses mediante entrevistas telefónicas estructuradas y se revisaron las bases de datos de los hospitales para evaluar si posteriormente presentaron HSA.

Para el diagnóstico de HSA, se consideró la presencia de cualquiera de los siguientes criterios: (1) Sangre en el espacio subaracnoideo a la evaluación mediante TCC. (2) Xantocromía en el líquido cefalorraquídeo a la inspección visual luego del centrifugado. (3) Detección de más de 5 glóbulos rojos en el líquido cefalorraquídeo y la presencia de un aneurisma demostrado mediante angiografía (tradicional, angio TC o angio RM). (4) Autopsia que confirme HSA. 
VALDEZ INTERNA:

¿Fue la muestra de pacientes representativa?

¿Fueron los pacientes suficientemente homogéneos con respecto al riesgo?

¿Fue el seguimiento suficientemente completo?

¿Se usaron criterios del outcome de forma objetiva e imparcial?

\section{ReSULTADOS:}

Se incluyeron 592 pacientes (59,1\% mujeres), con una edad promedio de 43 años. El seguimiento completo se realizó a 89,6\% de los pacientes. Las causas más frecuentes de cefalea en esta población correspondieron en primer lugar a cefaleas benignas, luego migraña y en tercer lugar HSA.

Tabla 1. Resultados de la estrategia diagnóstica

\begin{tabular}{|lrc|}
\hline & \multicolumn{2}{c|}{ Diagnóstico de HSA } \\
& $\mathrm{Si}$ & No \\
\hline Estrategia positiva & 61 & 175 \\
Estrategia negativa & 0 & 356 \\
\hline
\end{tabular}

Tabla 2. Sensibilidad, especificidad, valor predictivo negativo (VPN) y likelihood ratio (LR) de la estrategia diagnóstica

\begin{tabular}{|ll} 
Sensibilidad (95\% IC) & $100 \%(94-100)$ \\
Especificidad (95\% IC) & $67 \%(63-71)$ \\
VPN (95\% IC) & $100(98-100)$ \\
LR (+) & $3,03(2,69-3,53)$ \\
LR (-) & $<0,00001$
\end{tabular}

\section{COMENTARIOS Y APLCACIÓN PRÁCTICA:}

Si bien el trabajo enfoca un tema diagnóstico, los autores realizaron un estudio de cohorte prospectiva evaluando el riesgo de desarrollar una HSA en aquellos pacientes con una estrategia diagnóstica negativa, por lo que se debe analizar con criterios de validez para estudios de pronóstico (cohorte).

El tiempo de seguimiento (entre 6 a 36 meses) parece clínicamente razonable, pero no se logró seguir 10,4\% de los pacientes. Aunque se revisaron los registros de los hospitales para descartar que ellos hubiesen tenido una hospitalización por HSA, estos pacientes no fueron entrevistados por lo que no se puede descartar una evolución desfavorable. Esto podría introducir sesgo, pues si asumimos el peor escenario y todos hubiesen presentado una HSA el LR (-) habría tenido un valor de 0,7 que es un pésimo resultado y no permitiria descartar la patología.

Los pacientes evaluados son semejantes a aquellos que pueden consultar en un Servicio de Ungencia de nuestro medio, y la evaluación que se les realiza es reproducible en nuestro contexto. 
Dado los resultados, en pacientes con cefalea aguda no traumática, con PL y TCC negativos sería posible descartar la presencia de HSA aunque los resultados deben ser tomados con cautela pues el bajo LR (-) podría estar sobreestimado.

\section{REFERENCIAS}

1. Perry JJ, Stiel IG, Weus GA. Historical cohort study "use and yield of investigations for alert patients with possible subarachnoid hemorrhage". Can J Emerg Med 2002; 4: 333-37.

2. HaUerberg J, ANDERSEN BB, EsKesen V. Importance of the recognition of a warning leak as a sign of a ruptured intracranial aneurysm. Acta Neurol Scand 1991; 83: 61-4.

3. PolmEar A. Sentinel headaches in aneurysmal subarachnoid haemorrhage: what is the true incidence? A systematic review. Cephalalgia 2003; 23: 935-41.

4. At-Shaht R, White PM, Davenport RJ, Lndsay KW. Subarachnoid haemorhage. BMJ 2006; 333: 235-40. 